\title{
Chromosome and DNA methylation dynamics during meiosis in the autotetraploid Arabidopsis arenosa
}

\author{
Ana Carvalho · Margarida Delgado · Augusta Barão • \\ Márcia Frescatada · Edna Ribeiro · Craig S. Pikaard • \\ Wanda Viegas · Nuno Neves
}

Received: 23 February 2009/Accepted: 22 August 2009/Published online: 16 September 2009

(C) Springer-Verlag 2009

\begin{abstract}
Variation in chromosome number due to polyploidy can seriously compromise meiotic stability. In autopolyploids, the presence of more than two homologous chromosomes may result in complex pairing patterns and subsequent anomalous chromosome segregation. In this context, chromocenter, centromeric, telomeric and ribosomal DNA locus topology and DNA methylation patterns were investigated in the natural autotetraploid, Arabidopsis arenosa. The data show that homologous chromosome recognition and association initiates at telomeric domains in premeiotic interphase, followed by quadrivalent pairing of ribosomal 45S RNA gene loci (known as NORs) at leptotene. On the other
\end{abstract}

Communicated by Scott Russell.

A. Carvalho and M. Delgado contributed equally to this work.

A. Carvalho $\cdot$ M. Delgado $(\bowtie) \cdot$ A. Barão $\cdot$ M. Frescatada .

E. Ribeiro $\cdot$ W. Viegas $\cdot$ N. Neves

Centro de Botânica Aplicada à Agricultura, Instituto Superior

de Agronomia, Technical University of Lisbon,

Tapada da Ajuda, 1349-017 Lisbon, Portugal

e-mail: mxdelgado@isa.utl.pt

M. Delgado

Faculdade de Engenharias e Ciências Naturais,

Universidade Lusófona de Humanidades e Tecnologias,

Campo Grande 376, 1749-042 Lisbon, Portugal

C. S. Pikaard

Biology Department, Washington University,

St. Louis, MO 63130, USA

N. Neves

Departamento de Ciências da Vida, Faculdade de Ciências

e Tecnologia, Universidade Nova de Lisboa,

Campus da Caparica, 2829-516 Caparica, Portugal hand, centromeric regions at early leptotene show pairwise associations rather than associations in fours. These pairwise associations are maintained throughout prophase $\mathrm{I}$, and therefore likely to be related to the diploid-like behavior of $A$. arenosa chromosomes at metaphase I, where only bivalents are observed. In anthers, both cells at somatic interphase as well as at premeiotic interphase show 5-methylcytosine (5-mC) dispersed throughout the nucleus, contrasting with a preferential co-localization with chromocenters observed in vegetative nuclei. These results show for the first time that nuclear distribution patterns of 5-mC are simultaneously reshuffled in meiocytes and anther somatic cells. During prophase I, 5-mC is detected in extended chromatin fibers and chromocenters but interestingly is excluded from the NORs what correlates with the pairing pattern.

Keywords Meiosis - Autopolyploid D DNA methylation · Arabidopsis arenosa

\section{Introduction}

Polyploidy frequently accompanies speciation and is a key evolutionary mechanism affecting the life history of most eukaryotes, especially plants. There are two different types of polyploidy. Autopolyploidy refers to having more than one diploid chromosome set from a single species' genome. By contrast, allopolyploidy is the result of multiple genomes being united in a single nucleus (Corredor et al. 2007). Either type of polyploidization can result in the emergence of genotypic plasticity, providing polyploids with the potential to tolerate mutations or adapt duplicate genes for new functions more readily than diploids (Hegarty and Hiscock 2008). 
Contrary to animals, where meiocytes derive from differentiation of prior designated primordial germ cells, meiosis in plants defines the transition from the diploid sporophyte to the haploid gametophyte. Despite the different origin of meiocytes, however, the meiotic process is highly conserved in animals and plants, involving a common set of genes responsible for homologous chromosome pairing and recombination and chromosome segregation such that metaphase I of diploids is characterized by the presence of bivalents (Bozza and Pawlowski 2008). Meiotic analysis of diploid $A$. thaliana showed that telomeric domains direct homologous pairing, beginning at premeiotic interphase, whereas centromeric domains pair at zygotene (Armstrong and Jones 2003).

Numerous studies ranging from yeasts to plants show that polyploidy often affects meiotic behavior, namely, pairing patterns, recombination rates and chromosome segregation (Santos et al. 2003; Storchová et al. 2006; Corredor et al. 2007). In particular, due to the presence of more than two homologous (autopolyploid) or homoeologous (allopolyploid) chromosome sets, multivalents are often formed at metaphase I (Sybenga 1996; Naranjo and Corredor 2008). Multivalent pairing often results in disturbed chromosome segregation and reduced fertility. To overcome this problem, many polyploids have evolved genetic mechanisms that result in a diploid-like behavior at metaphase I (Naranjo and Corredor 2008). Such genomic adaptations illustrate the importance of meiosis in evolutionary biology, since many recognized cases of diploid genomes have undergone ancestral genome duplication (Mitchell-Olds and Clauss 2002; Clauss and Koch 2006).

The genus Arabidopsis includes natural autopolyploids, yet despite the emergence of Arabidopsis thaliana as a model for studies of genome restructuring and speciation, investigation on meiotic behavior in Arabidopsis polyploid species is sparse (Bomblies and Weigel 2007). Comparison between natural and synthetic polyploids has been examined for tetraploid lines of A. thaliana (Santos et al. 2003) and the allotetraploid of $A$. thaliana and A. arenosa, known as A. suecica (Comai et al. 2003). These data show that in synthetic polyploids, multivalents outnumber bivalents during metaphase I, contrasting with equivalent numbers of bivalents and multivalent in a natural autotetraploid (Santos et al. 2003). However, in the synthetic A. suecica, homologous paring was reached as early as three generations after synthesis (Comai et al. 2003). These results indicate that partial diploidization seems to be progressively established, as proposed by Weiss and Maluszynska (2000).

It has been shown that DNA methylation mediates the silencing of alleles from imprinted genes in plants and animals (Scott and Spielman 2006). In Arabidopsis, the distribution pattern of 5-methylcytosine $(5-\mathrm{mC})$ alters in response to developmental cues. Although $5-\mathrm{mC}$ is generally restricted and highly concentrated at chromocenters in somatic interphase nuclei (Fransz et al. 2006), this pattern is not static. Alterations include evidently dispersed 5-mC residues at specific developmental stages, namely, immediately after germination and during floral transition (Pontes et al. 2007; Tessadori et al. 2007).

In this study, meiosis was characterized in Arabidopsis arenosa $(2 n=4 x=32)$, thereby exploiting an autotetraploid Arabidopsis species found in nature that together with A. thaliana is one of the progenitors of the model allopolyploid, A. suecica. Analysis of telomeric domains, nucleolus organizing regions (NORs; 45S ribosomal RNA gene clusters) and centromeric regions as well as the distribution patterns of 5-mC show that telomeric associations initiate meiotic chromosome pairing, followed by quadrivalent NORs pairing. In contrast, pairing of centromeric domains is restricted to pairs throughout prophase I, which is in accord with the diploid-like behavior observed in metaphase I.

\section{Materials and methods}

\section{Plant material}

The natural autopolyploid Arabidopsis arenosa $(2 n=$ $4 x=32$ ) (Arabidopsis Biological Resource Center stock CS3901) was grown in a greenhouse (16 $\mathrm{h}$ photoperiod at $\left.22 \pm 2{ }^{\circ} \mathrm{C}\right)$. Whole inflorescences and roots were fixed in ethanol/acetic acid $(3: 1 \mathrm{v} / \mathrm{v})$ and stored at $-20^{\circ} \mathrm{C}$. Anthers were isolated and spreads were performed after enzymatic digestion as previously described (Armstrong et al. 2001). Stage identification of meiocytes in this study follow the description presented for Arabidopsis thaliana by Ross et al. (1996), distinguishing early from later prophase I stages through the grouping of chromosomes into a synizetic knot and through the further dispersion of cytoplasmic organelles throughout the cell. Chromosome morphology allowed for the characterization of each stage of prophase I, classifying cells presenting extended, tenuous and intertwined single chromosomes as leptotene and those already showing some synapsed regions and the characteristic synizetic knot as zygotene. Pachytene cells were characterized by the absence of asymmetric distribution of organelles and obvious chromosome differentiation due to full alignment of homologous chromatids at synapsis. Other meiotic stages were identified based on consensus descriptions of meiotic development in other angiosperms (Ross et al. 1996). 
DNA in situ hybridization and immunolocalization of 5-methylcytosine

DNA fluorescence in situ hybridization (FISH) was performed using probes for the following sequences: pAaCEN for centromeric DNA (CEN), telomeric DNA (TEL) and ribosomal DNA (NOR). Probes for centromeric (pAaCEN) and ribosomal DNA (pCaIGS) were obtained as described in Pontes et al. (2004) and labeled through incorporation of digoxigenin-dUTP or biotin-dUTP. The telomeric DNA probe was obtained by PCR (Ijdo et al. 1991) using oligonucleotides (TTTAGGG) 5 and (CCCTAAA) $)_{5}$, corresponding to the Arabidopsis telomere sequence, in the presence of biotin-dUTP.

Hybridization conditions, stringency washes and detection of hybridization sites followed the procedure of Pontes et al. (2004). Biotinylated probes and probes labeled with digoxigenin were detected with streptavidin-Cy3 (1:200, Sigma) and anti-digoxigenin-FITC (1:10, Roche), respectively. Nuclei were counterstained with DAPI $(1 \mathrm{mg} / \mathrm{ml})$ diluted in Citifluor (Agar).

Immunodetection of 5-mC was performed prior to FISH as described in Pontes et al. (2007) using monoclonal antibody against 5-mC (1:200, AB10805-50 Abcam) and detected with anti-mouse-Cy3 IGG (1:100, Sigma).

The number of DAPI-positive chromocenters and DNA hybridization sites was scored.

Fluorescence signals were analyzed using an epifluorescence microscope Zeiss Axioskop2 equipped with a Zeiss AxioCam MRc5 digital camera. Images were captured separately for each fluorochrome using the appropriate excitation and emission filters and merged using
Adobe Photoshop 7.0 (Adobe Systems) software, DAPI images were converted to gray scale that allow better visualization of chromatin details.

\section{Results}

In diploid A. thaliana, as in other Arabidopsis species, interphase chromatin organization is characterized by the presence of chromocenters cytologically defined as condensed chromatin domains that display strong DAPI staining. Chromocenters are defined in a molecular sense as domains that comprise the heterochromatic fraction of the genome and include centromeric and ribosomal DNA chromatin (Fransz et al. 2002, 2006). The organization of A. arenosa chromocenters analyzed by DAPI staining revealed these genomic domains to be discrete heterochromatic knobs in somatic anther cells and at premeiotic interphase (PMI). The centromeric domains observed by fluorescent hybridization with pAaCEN probe show that centromeric sequences are mostly condensed, coincident with chromocenters, and widely dispersed throughout the nucleus during PMI (Fig. 1). At PMI, an average number of 32.2 chromocenters and 29.2 signals of centromeric DNA are visible per nucleus (Table 1). Both chromocenter and centromeric domain mean scores approximate the chromosome number of $A$. arenosa $(2 n=4 x=32)$. The average number of centromeric domains is lower than the chromosome number as a result of infrequent centromeric association between chromosomes. Nuclei were assayed for relative positioning of centromeric and chromocenter domains, with $98.5 \%$ co-localization of chromocenter and
Fig. 1 Interphase and prophase somatic cells from anthers after FISH with pericentromeric DNA (CEN), and premeiotic interphase nuclei (PMI) after FISH with pericentromeric DNA (CEN), ribosomal DNA (NOR) telomere DNA (TEL). For each nucleus, DAPI staining is shown in the top row and hybridization signals in the bottom row, central row shows the merge of both images. All images have identical magnification and bar $5 \mu \mathrm{m}$

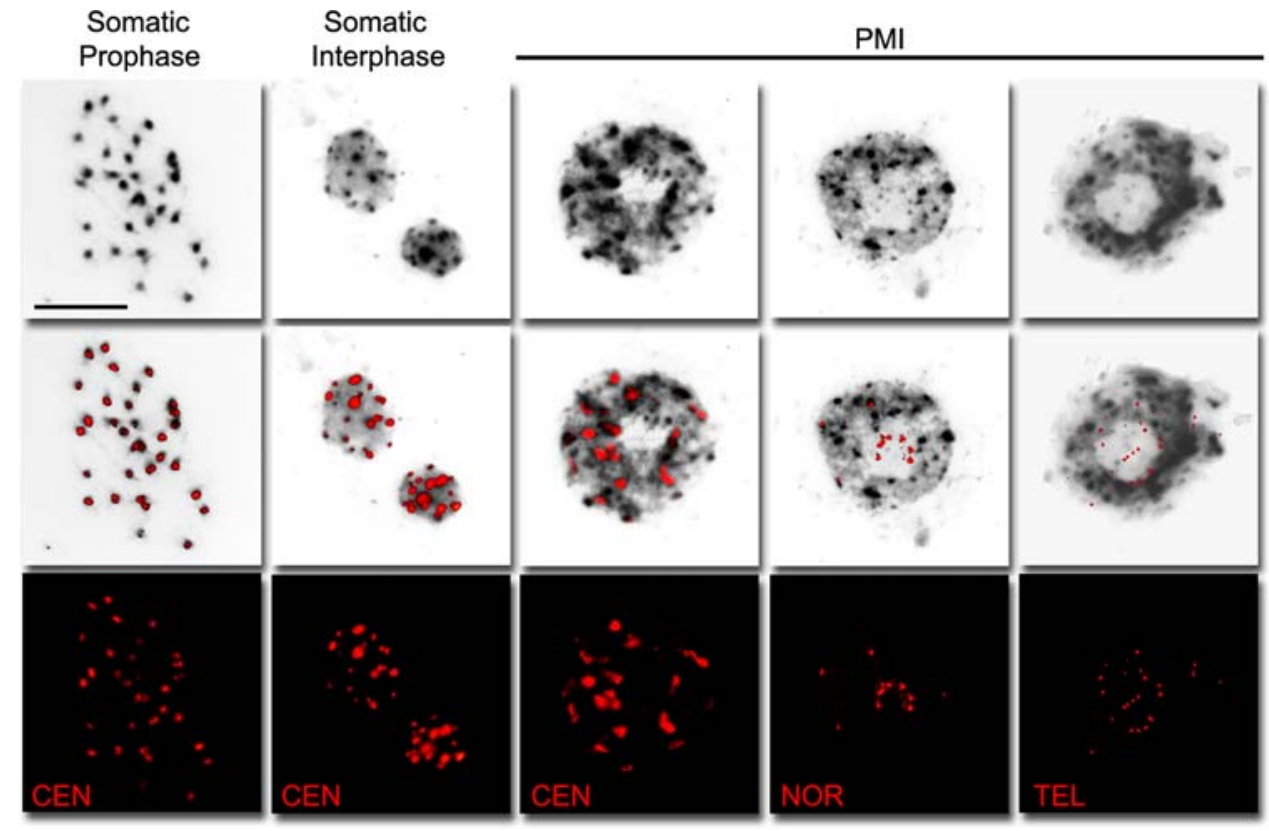


Table 1 Average number ( \pm standard error) of DAPI-positive chromocenters (CC) and hybridization signals for centromeric DNA (CEN), ribosomal DNA (NOR) and telomeric DNA (TEL) in meiocytes

\begin{tabular}{lllllrrrr}
\hline & CC & $n^{*}$ & \multicolumn{1}{l}{ CEN } & $n^{*}$ & \multicolumn{1}{l}{ NOR } & $n^{*}$ & TEL & $n^{*}$ \\
\hline PMI & $32.2( \pm 3.42)$ & 47 & $29.2( \pm 3.06)$ & 26 & $10.0( \pm 2.45)$ & 21 & $21( \pm 2.00)$ & 22 \\
Leptotene & $19.4( \pm 2.75)$ & 56 & $14( \pm 3.13)$ & 32 & $2.9( \pm 0.85)$ & 24 & $18( \pm 2.53)$ & 24 \\
Zygotene & $14.4( \pm 2.68)$ & 51 & $11.8( \pm 1.58)$ & 21 & $2.6( \pm 0.77)$ & 30 & $16( \pm 2.64)$ & 24 \\
Pachytene & $12.7( \pm 2.08)$ & 50 & $11.5( \pm 2.71)$ & 25 & $2.6( \pm 0.92)$ & 25 & $16( \pm 2.67)$ & 25 \\
\hline
\end{tabular}

$n *$ represents the number of cells analyzed

centromeric signals observed in PMI nuclei, which is expected based on the dominant contribution of centromeric repeats to chromocenters (Pontes et al. 2004). In turn, DAPI-stained domains exceeded the chromosome number in some cells. This likely resulted from $45 \mathrm{~S}$ rDNA loci (NORs) signals that adopt (in part or in whole) a compact configuration as DAPI-positive centromere-like containing chromocenters (Pontes et al. 2004).

Considering the NORs, which along with the centromeric domains compose much of the heterochromatin fraction in Arabidopsis species, their meiotic organization was examined by in situ hybridization with a DNA probe specific for A. arenosa rDNA (pAaIGS) (Fig. 1). In PMI cells of A. arenosa, we observed an average number of 10.0 hybridization sites, although 12 NORs are present per nucleus. The lower observed number is apparently due to NOR association (Fig. 1). In fact, in PMI cells, NORs frequently associate near the nucleolar boundary. In addition, it was possible to observe compact as well as decondensed rDNA arrays.

At PMI, detection of telomeres with the telomeric DNA probe showed that these chromosome domains are mostly clustered at the nucleolus at this stage (Fig. 1), as was also observed in the diploid A. thaliana (Armstrong and Jones 2003). Additionally, by scoring the number of in situ signals for telomere domains (Table 1), an average of 21 signals per PMI cell are visible, indicating a marked association of telomeric regions just before prophase I initiates. This observation agrees with the analysis of meiosis in the diploid, A. thaliana (Armstrong and Jones 2003). A diploid-like telomere pairing in A. arenosa would yield 32 in situ signals, and a tetraploid pairing would result in 16 signals.

Telomere and NOR show a quadrivalent paring at prophase I

During prophase I, the crucial meiotic processes of homologous chromosome recognition, pairing, alignment and recombination take place. Prophase I meiocytes were identified based on distribution of cell organelles, nucleolus positioning and chromatin conformation, following descriptions of Ross et al. (1996) and Armstrong and Jones (2003) (see "Materials and methods"). Meiocytes at leptotene show characteristic stretches of unsynapsed chromosome axes. Considering telomeric domains, there is a progressive reduction in the mean number of in situ signals from PMI to leptotene and zygotene, where 18.0 and 16.0 telomere signals were observed per cell, respectively (Fig. 2a, LEP, ZYG; Table 1), a number maintained until pachytene (Fig. 2a, PACH). This observation confirms an association of telomeric domains from four homologous chromosomes. Considering telomere nuclear distribution, clustering of telomeric domains around the nucleolus was frequently maintained from PMI to leptotene. By zygotene, and later at pachytene, that nucleolar disposition is no longer present (Fig. 2a).

In agreement with telomeric analyses that point to a quadrivalent pairing of $A$. arenosa chromosomes, the rDNA loci also synapse in groups of four, such that the 12 NORs present are seen as three hybridization signals in leptotene meiocytes; this pairing number is maintained throughout zygotene and pachytene (Fig. 2b, LEP, ZYG and $\mathrm{PACH}$; Table 1). In diplotene/diakinesis, higher numbers of in situ rDNA signals were observed (4-6 signals per cell) (Fig. 2b, DIP/DIAK), revealing that quadrivalent pairing structures are resolved (Fig. 2b, METI, ANAPH I). Regarding the organization of ribosomal chromatin, the $45 \mathrm{~S}$ rDNA loci show a condensed pattern throughout prophase I.

A diploid-like behavior is observed for centromeric domains during prophase I and for chromosomes at metaphase I and anaphase I

Leptotene cells of $A$. arenosa probed for centromeric chromatin domains show a drastic diminution of the centromeric number: from 29.2 at PMI to 14.0 (Fig. 3, LEP; Table 1). The reduction in the number of observed centromeric domains is accompanied by similar diminution of the average number of chromocenters (from 32.2 signals at PMI to 19.4 at leptotene, Table 1). Given that a regular pairwise association of centromeric domains would result in 16 centromeric in situ signals, the results indicate that 
A

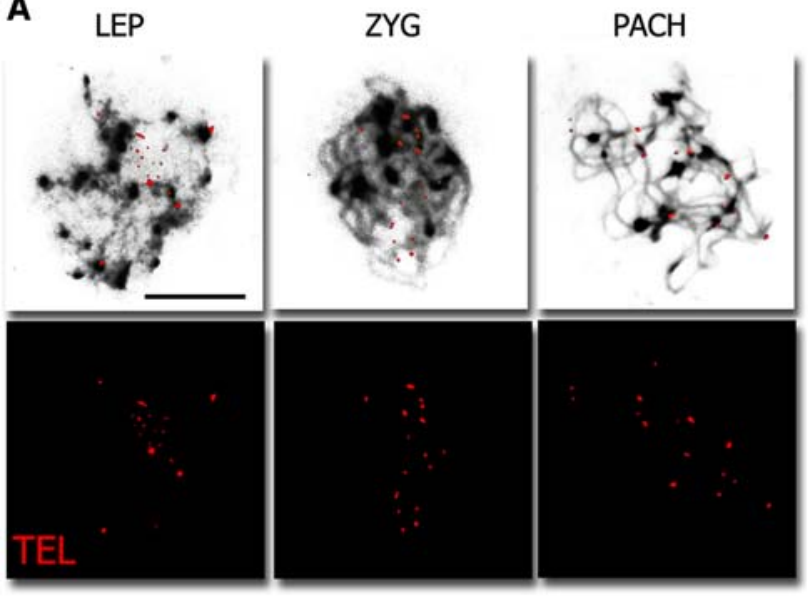

B

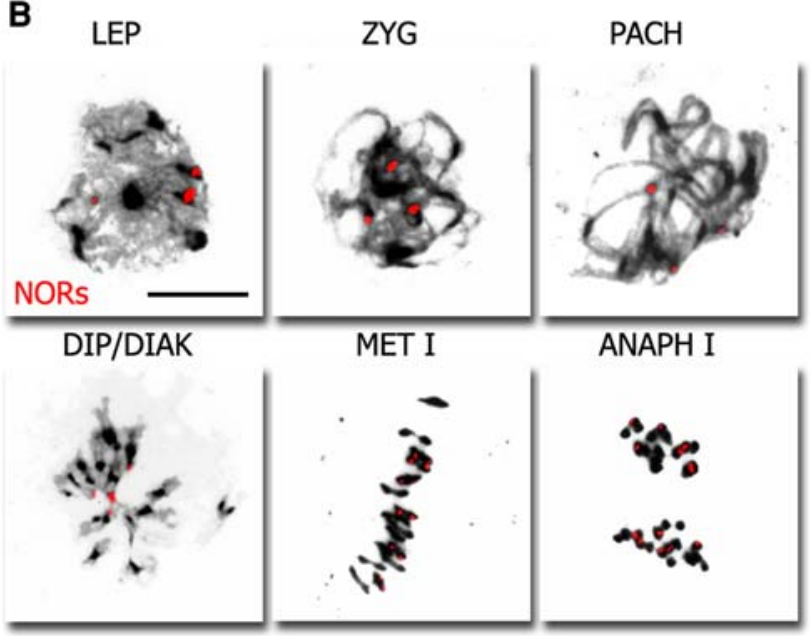

Fig. 2 Meiocytes in distinct stages of prophase I after FISH with a telomere DNA (TEL) and b ribosomal DNA (NOR). In a, top row shows merge images of telomere hybridization signals and DAPI staining for meiocytes at leptotene (LEP), zygotene (ZYG) and pachytene $(\mathrm{PACH})$; in the bottom row images of hybridization signals alone are presented. In $\mathbf{b}$, merge images of NOR hybridization signals and DAPI staining are presented for meiocytes at leptotene (LEP), zygotene $(\mathrm{ZYG})$, pachytene $(\mathrm{PACH})$, diplotene/diakinesis (DIP/ DIAK), metaphase I (MET I) and anaphase I (ANAPH I). All images have identical magnification and bar $5 \mu \mathrm{m}$

these domains associate in pairs at prophase I. Therefore, the pairing in fours initiated by telomeric domains and extended to adjacent loci as NORs does not rule pairing of centromeric domains that are largely restricted to a pairwise manner. In addition, pairing of these domains occurs at the beginning of prophase I, earlier than that described for A. thaliana (Armstrong et al. 2001).

The zygotene stage in A. arenosa (Fig. 3, ZYG) is characterized by 12 centromeric domain signals per cell, similar to that observed at pachytene (Fig. 3, PACH). Equivalent reduction in the number of signals was detected for chromocenters (Table 1). Pachytene chromosomes are mainly visible as fully paired double structures revealed by the thickening of the thread-like chromosomes observed during zygotene (Fig. 3, PACH; see also Figs. 1, 2, $\mathrm{PACH})$.

After pachytene, chromocenters adopt a more defined and compact structure with an average number of 15.5 DAPI-positive domains per diakinesis meiocyte, reinforcing the diploid-like behavior later observed (Fig. 2, DIP/ DIAK).

Metaphase I and anaphase I stages are characterized by the absence of nucleolus and nuclear envelope, and maximum chromatin condensation, leading to a loss of discrimination between euchromatic and heterochromatic domains. Tetraploid A. arenosa meiocytes analyzed by DAPI staining at metaphase I show 16 bivalents aligned at the spindle plate, with most bivalents in an open (rod) configuration (Fig. 3, MET I) corresponding on average to one chiasma per pair of homologous chromosomes. These results are indicative of low recombination rates, since only occasionally some cells showed two to four closed (ring) bivalents (see Fig. 2b, MET I for example); no multivalents or univalents were detected. This clearly points to a highly regular diploid behavior of tetraploid A. arenosa chromosomes at this meiotic stage. The stability of the meiotic process is further confirmed during anaphase I chromosome segregation, where 16 chromosomes (and 16 centromeric domains) are observed per pole (Fig. 2b, ANAPH I).

In A. arenosa meiocytes at metaphase I, 12 condensed NORs are detected, with homologous loci from each pair facing opposite poles of the plate (Fig. 2b, MET I). At anaphase I, ribosomal chromatin compaction states are maintained and six signals are observed per haploid chromosome group (Fig. 2b, ANAPH I), consistent with a diploid-like organization in the tetraploid A. arenosa at this meiotic stage.

DNA methylation is a dynamic epigenetic mark at $A$. arenosa meiosis

To investigate if the developmental shift associated with meiosis is accompanied by changes in DNA methylation patterns, we performed immunodetection of 5-methylcytosine $(5-\mathrm{mC})$ residues in anther somatic and meiotic cells. Considering that centromeric domains and NORs represent much of the heterochromatic fraction, and given that the two have distinct pairing and chromatin conformation patterns during prophase I, simultaneous in situ hybridization to rDNA loci and immunolocalization of 5-mC was also performed. Contrary to what is observed in vegetative tissues, foci of methylated DNA are spread over the euchromatic and heterochromatic fractions in anther somatic and premeiotic interphase cells of A. arenosa (Fig. 4a). 
Fig. 3 Meiocytes after FISH with pericentromeric DNA (CEN) at leptotene (LEP), zygotene (ZYG), pachytene $(\mathrm{PACH})$, diplotene/diakinesis (DIP/DIAK), metaphase I (MET I) and anaphase I (ANAPH I). For each nucleus, DAPI staining is shown in the top row and hybridization signals in the bottom row; central row shows the merge of both images. All images have identical magnification, bar $5 \mu \mathrm{m}$

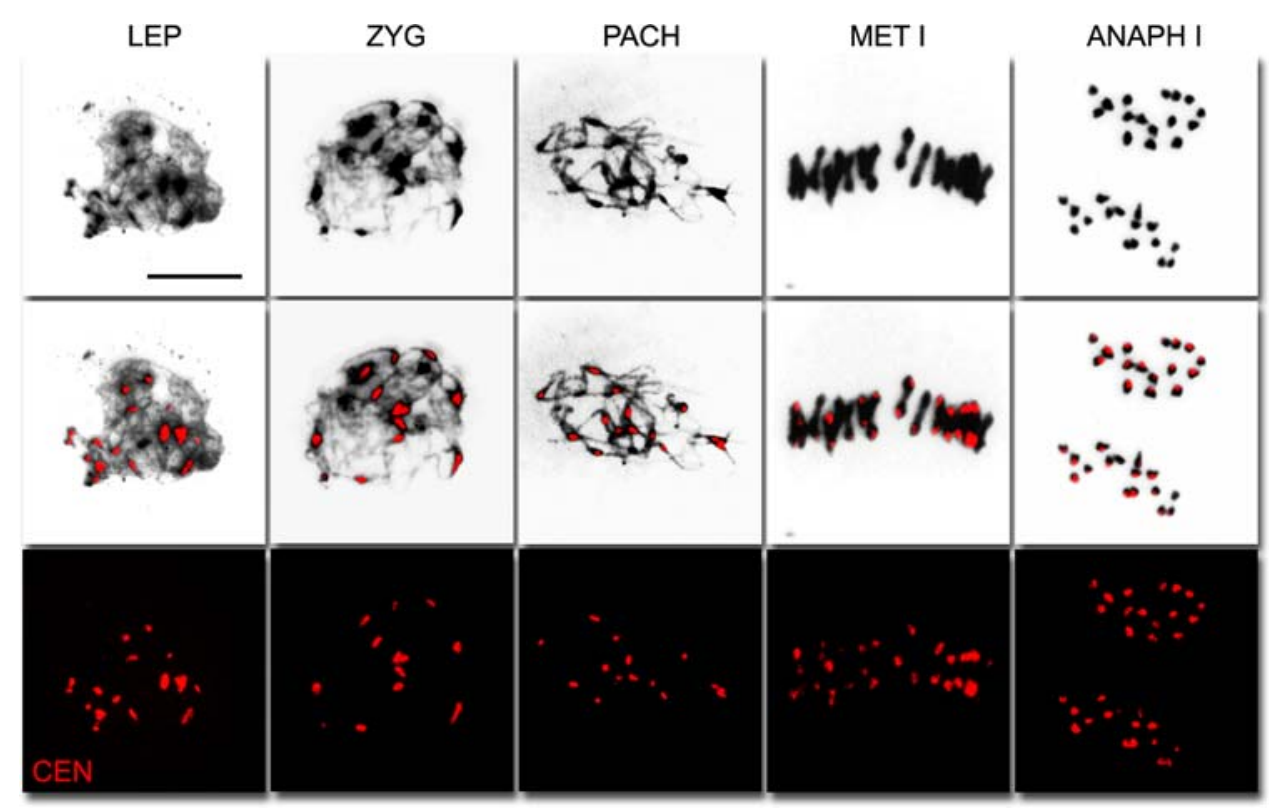

From leptotene to pachytene, strong immunosignals of 5-mC are detected in nuclear domains corresponding to chromocenters, although labeling is also evident on extended chromosome fibers that emanate from the chromocenters (Fig. 4b, LEP, ZYG and PACH). However, more evident in pachytene chromatin is that some paired domains show the presence of 5-mC, whereas other chromatin fibers are virtually free of methylated cytosines (Fig. 4b, PACH). The presence of 5-mC residues in chromosome arms and its absence in centromeric regions is clearly visualized at metaphase I, where 5-mC signals are present throughout all bivalents, with the exception of the centromeres (Fig. 4b, MET I, arrows). At anaphase I, the extreme chromosome compaction renders it impossible to distinguish between chromocenters and chromosome arms, and 5-mC labeling mainly coincides with DAPI staining (Fig. 4b, ANAPH I). However, in prophase II, 5-mC is observed throughout the nuclear area although with stronger intensity at chromocenters (Fig. 4b, PROPH II). Interestingly, rDNA loci seem depleted of methylated cytosines, since no overlap between methylated domains and NORs is observed throughout prophase I (Fig. 4b, bottom row LEP, ZIG and PACH).

\section{Discussion}

In the present work, we present for the first time a detailed analysis of the meiotic chromosome behavior of the natural autotetraploid Arabidopsis arenosa $(2 n=4 x=32)$ and establish a correlation between chromatin dynamics and the particular aspects of meiosis I. Meiotic metaphase I of A. arenosa is characterized as having a perfect diploid-like behavior, with visible bivalents and total absence of multivalents. The large prevalence of open (rod) bivalents representing one chiasma indicate moreover low recombination rates in A. arenosa. A higher rate of homologous recombination was described in A. thaliana, where four to five closed (ring) bivalents are observed at metaphase I, i.e. almost all bivalents are closed (Sanchez-Moran et al. 2002; Armstrong and Jones 2003; Santos et al. 2003).

Our results indicate that in the autotetraploid A. arenosa, telomeric domains tend to associate in fours during early prophase I. Telomere association is initiated at premeiotic interphase and is strengthened from leptotene to zygotenepachytene. Furthermore, telomeric regions are noticeably associated at nucleolar boundaries in premeiotic interphase nuclei and at early prophase I, in accordance with observations of somatic interphase nuclei from meristematic root cells of diploid A. thaliana (Fransz et al. 2002). Homologous association of $A$. thaliana telomeric domains was also detected at leptotene by Armstrong et al. (2001). Our results of telomere distribution in the autotetraploid A. arenosa are therefore consistent with the conclusions drawn from diploid A. thaliana, revealing that telomere clustering allows homologous recognition and directs correct pairing in Arabidopsis independently of ploidy/dosage level. However, this association appears to occur earlier in A. arenosa (preleptotene) than in A. thaliana.

The increasing thickness of chromatin fibers observed from leptotene to zygotene indicates an association of homologous $A$. arenosa chromosomes, further supported by the analysis of rDNA loci. In A. thaliana, there is a strong variability in the number of in situ rDNA signals at early prophase I (Yang et al. 2006), demonstrating the occurrence of non-homologous NOR association. In A. arenosa 


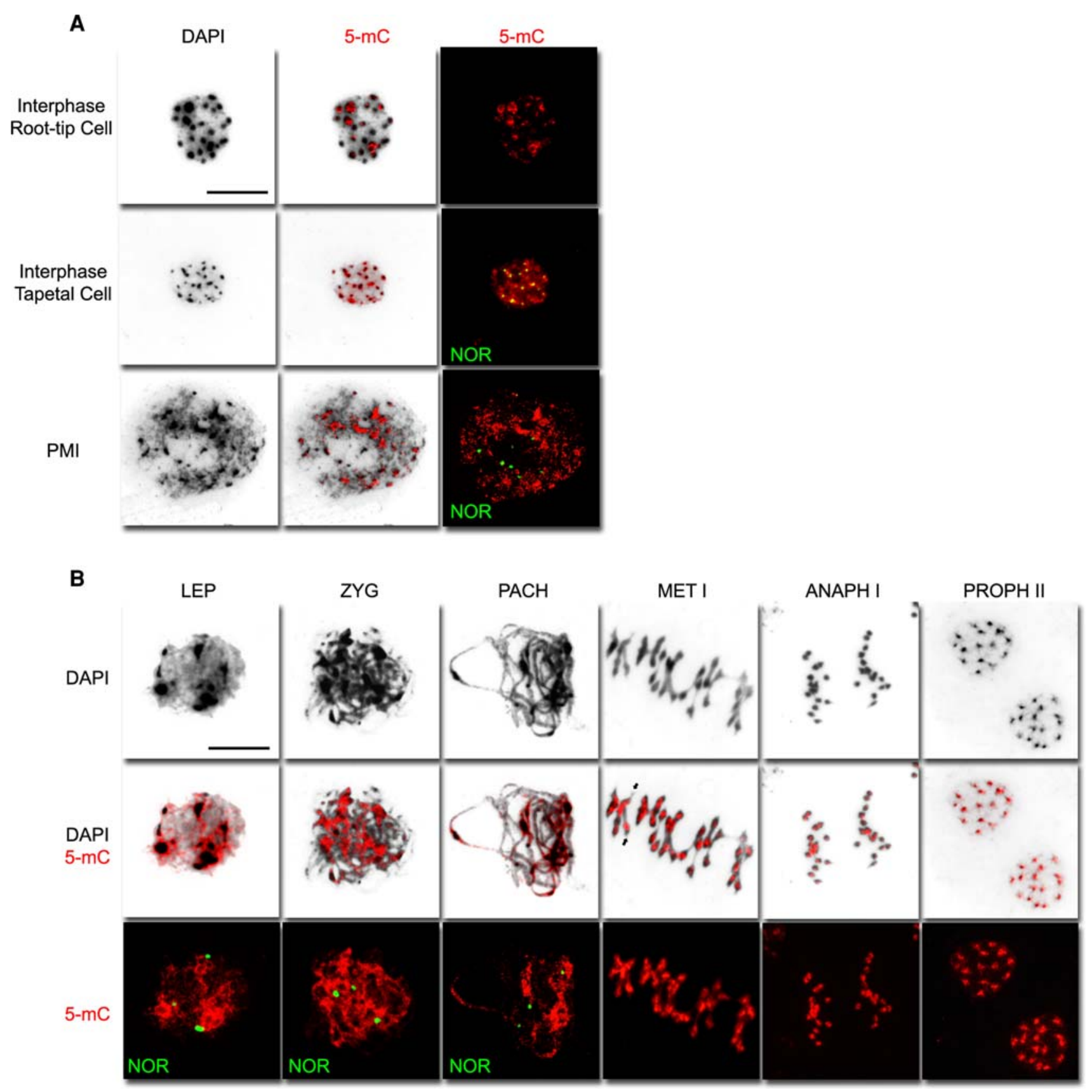

Fig. 4 5-Methylcytosine (5-mC) distribution patterns in root tip and anther cells. a Interphase nucleus from root tip cell after immunodetection of 5-methylcytosine (5-mC, red); nuclei from anther somatic interphase (tapetal cell) and from meiocyte at premeiotic interphase (PMI) after simultaneous immunodetection of 5-methylcytosine (5-mC, red) and FISH with rDNA (NOR, green). For each nucleus, DAPI staining is shown in the left column, merge images of DAPI staining and 5-mC signals in the central column and 5-mC NOR signals in the right column. b Meiocytes at leptotene (LEP), zygotene (ZYG), pachytene (PACH), metaphase I (MET I), anaphase

meiocytes, however, variability in the number of in situ rDNA signals per cell is nearly nonexistent. Paralleling this fact, striking differences in rDNA organization are also
I (ANAPH I) and prophase II (PROPH II) after immunodetection of 5-methylcytosine (5-mC, red). For pachytene (PACH), FISH with rDNA is also shown (NOR, green, bottom panel). For each nucleus, DAPI staining is shown in the top row, merge images of DAPI staining and 5-mC signals in the central row and 5-mC images in the bottom row. From leptotene to metaphase I regions of reduced 5-mC signal intensity corresponding to the central domain of centromeric chromocenters are visible and exemplified by arrows for one bivalent at metaphase I. All images have identical magnification, bar $5 \mu \mathrm{m}$

observed between A. thaliana and A. arenosa during early stages of prophase I. In A. thaliana meiocytes, the in situ signals for NORs show marked decondensation from 
leptotene to pachytene (Yang et al. 2006). In contrast, rDNA signals are discrete and show strong compaction from leptotene to pachytene in A. arenosa.

In contrast to telomeric and NOR domains, A. arenosa centromeric domains are mostly associated in pairs during early prophase I. There is therefore reduced synapsis of those domains in A. arenosa compared to the diploid A. thaliana, where the number of centromeric FISH signals decreases from 10 at leptotene to an average number of 2.4 in zygotene (where 5 signals were expected as a result of diploid pairing) (Armstrong et al. 2001; Armstrong and Jones 2003). As observed for the pairing of telomeric domains, there are also temporal differences in centromeric domains pairing between A. arenosa and A. thaliana. In A. arenosa, pairing is established at early leptotene, whereas in A. thaliana, chromocenters pair at mid zygotene (Ross et al. 1996; Armstrong et al. 2001; Armstrong and Jones 2003).

The functional role of centromeric sequences in homologous recognition and pairing has been widely discussed, with conflicting results in different organisms. In A. thaliana, centromeric associations at prophase I are preceded and directed by the telomeres (Armstrong and Jones 2003), as was also recently shown for allohexaploid wheat (Corredor et al. 2007). Both in wheat and in diploid A. thaliana, there is evidence that centromeric associations detected at early prophase I occur frequently between non-homologous chromosomes, while telomere pairing occurs between homologous chromosomes. In A. arenosa, our data is also consistent with telomere pairing preceding further events in prophase I, but suggests an orderly pairing of centromeric domains at early prophase I.

In our cytogenomic approach, DNA methylation patterns were characterized throughout meiosis in A. arenosa. The results are compatible with a major reshuffling of DNA methylation in reproductive tissues. Globally, one of the most striking lines of evidence for changes in DNA methylation comes from 5-mC distribution patterns in interphase anther cells (somatic and premeiotic) when compared to vegetative interphase patterns observed in A. arenosa, and also with those previously described in other Arabidopsis species (Fransz et al. 2006 for A. thaliana; Pontes et al. 2007 for $A$. suecica). In interphase nuclei observed in vegetative tissues of A. thaliana, highly methylated DNA domains are physically confined to the chromocenters (Fransz et al. 2006), with centromeric domains showing a central region with diminished cytosine methylation corresponding to the centromere, where CenH3 is deposited (Zhang et al. 2008). In contrast, in anther somatic as well as premeiotic interphase nuclei of A. arenosa, we observed 5-mC immunosignals dispersed throughout the nuclear area.
The fact that in A. suecica, the allopolyploid between A. thaliana and A. arenosa, the nucleolar dominance characteristic of vegetative organs is erased in the transition from the inflorescence to floral meristem (Chen and Pikaard 1997), a process that is mediated through DNA methylation (Preuss et al. 2008) suggests that the observed nuclear redistribution of $5-\mathrm{mC}$ in reproductive organs is a generalized process in Arabidopsis. A similar dispersal of 5-mC pattern was observed in A. thaliana rosette leaves during floral transition associated with chromocenter reduction (Tessadori et al. 2007). The inverse process, that is completion of chromocenter assembly, has been shown to occur between 2 and 4 days after seed germination both in A. thaliana (Mathieu et al. 2003) and A. suecica (Pontes et al. 2007). In this case $5-\mathrm{mC}$ is widely distributed throughout the nucleus in 2 days old plants and restricted to chromocenters in 4 days old plants (Pontes et al. 2007). Taken together, this indicates that major developmental cues are accompanied by epigenetic reshuffling mediated by DNA methylation.

As far as we know this is the first time that $5-\mathrm{mC}$ distribution throughout meiosis has been analyzed in plants, demonstrating that the onset of the reproduction phase is mediated by general and widespread alteration in the distribution of 5-mC.

In regard to chromosome segregation, our results clearly show a regular diploid behavior of $A$. arenosa. It can be argued that this might result from the fact that this natural autotetraploid might no longer be a polyploid and present already two distinct chromosome sets. However, association in fours of telomeres and NORs indicates that this is not fully achieved. During prophase I, methylated DNA is present in pericentric regions and chromosome arms and is totally absent in NORs. Taking into consideration that 5-mC positive pericentric domains do not show quadrivalent homologous pairing, it is tempting to suggest a potential correlation between the high density of that epigenetic mark and restriction to a pairwise association.

Acknowledgments We are grateful to $\mathrm{H}$. Sofia Pereira for the critical review. A.L. Carvalho and M. Delgado are funded by Fellowships (grants SRRH/BD/13830/03 and SFRH/BPD/27219/2006, respectively), and the work was financed by FCT/POCTI/59389/04 (P.I. N.Neves) from the Fundação para a Ciência e a Tecnologia.

\section{References}

Armstrong SJ, Jones GH (2003) Meiotic cytology and chromosome behaviour in wild-type Arabidopsis thaliana. J Exp Bot 54:1-10

Armstrong SJ, Franklin FC, Jones GH (2001) Nucleolus-associated telomere clustering and pairing precede meiotic chromosome synapsis in Arabidopsis thaliana. J Cell Sci 114:4207-4217

Bomblies K, Weigel D (2007) Arabidopsis: a model genus for speciation. Curr Opin Genet Dev 17:500-504 
Bozza CG, Pawlowski WP (2008) The cytogenetics of homologous chromosome pairing in meiosis in plants. Cytogenet Genome Res 120:313-319

Chen ZJ, Pikaard CS (1997) Transcriptional analysis of nucleolar dominance in polyploid plants: biased expression/silencing of progenitor rRNA genes is developmentally regulated in Brassica. Proc Natl Acad Sci USA 94:3442-3447

Clauss MJ, Koch MA (2006) Poorly known relatives of Arabidopsis thaliana. Trends Plant Sci 11:449-459

Comai L, Tyagi AP, Lysak MA (2003) FISH analysis of meiosis in Arabidopsis allopolyploids. Chromosome Res 11:217-226

Corredor E, Lukaszewski AJ, Pachón P, Allen DC, Naranjo T (2007) Terminal regions of wheat chromosomes select their pairing partners in meiosis. Genetics 177:699-706

Fransz P, De Jong JH, Lysak M, Castiglione MR, Schubert I (2002) Interphase chromosomes in Arabidopsis are organized as well defined chromocenters from which euchromatin loops emanate. Proc Natl Acad Sci USA 99:14584-14589

Fransz P, ten Hoopen R, Tessadori F (2006) Composition and formation of heterochromatin in Arabidopsis thaliana. Chromosome Res 14:71-78

Hegarty MJ, Hiscock SJ (2008) Genomic clues to the evolutionary success of polyploid plants. Curr Biol 18:R435-R444

Ijdo JW, Wells RA, Baldini A, Reeders ST (1991) Improved telomere detection using a telomere repeat probe (TTAGGG)n generated by PCR. Nucleic Acids Res 19:4780

Mathieu O, Jasencakova Z, Vaillant I, Gendrel AV, Colot V, Schubert I, Tourmente S (2003) Changes in 5S rDNA chromatin organization and transcription during heterochromatin establishment in Arabidopsis. Plant Cell 15:2929-2939

Mitchell-Olds T, Clauss MJ (2002) Plant evolutionary genomics. Curr Opin Plant Biol 5:74-79

Naranjo T, Corredor E (2008) Nuclear architecture and chromosome dynamics in the search of the pairing partner in meiosis in plants. Cytogenet Genome Res 120:320-330

Pontes O, Neves N, Silva M, Lewis MS, Madlung A, Comai L, Viegas W, Pikaard CS (2004) Chromosomal locus rearrangements are a rapid response to formation of the allotetraploid Arabidopsis suecica genome. Proc Natl Acad Sci USA 101: 18240-18245
Pontes O, Lawrence RJ, Silva M, Preuss S, Costa-Nunes P, Earley K, Neves N, Viegas W, Pikaard CS (2007) Postembryonic establishment of megabase-scale gene silencing in nucleolar dominance. PLoS ONE 2:e1157

Preuss SB, Costa-Nunes P, Tucker S, Pontes O, Lawrence RJ, Mosher R, Kasschau KD, Carrington JC, Baulcombe DC, Viegas W, Pikaard CS (2008) Multimegabase silencing in nucleolar dominance involves siRNA-directed DNA methylation and specific methylcytosine-binding proteins. Mol Cell 32:673-684

Ross KJ, Fransz P, Jones GH (1996) A light microscopic atlas of meiosis in Arabidopsis thaliana. Chromosome Res 4:507-516

Sanchez-Moran E, Armstrong SJ, Santos JL, Franklin FC, Jones GH (2002) Variation in chiasma frequency among eight accessions of Arabidopsis thaliana. Genetics 162:1415-1422

Santos JL, Alfaro D, Sanchez-Moran E, Armstrong SJ, Franklin FC, Jones GH (2003) Partial diploidization of meiosis in autotetraploid Arabidopsis thaliana. Genetics 165:1533-1540

Scott RJ, Spielman M (2006) Genomic imprinting in plants and mammals: how life history constrains convergence. Cytogenet Genome Res 113:53-67

Storchová Z, Breneman A, Cande J, Dunn J, Burbank K, O’Toole E, Pellman D (2006) Genome-wide genetic analysis of polyploidy in yeast. Nature 443:541-547

Sybenga J (1996) Chromosome pairing affinity and quadrivalent formation in polyploids: do segmental allopolyploids exist? Genome 39:1176-1184

Tessadori F, Schulkes RK, van Driel R, Fransz P (2007) Lightregulated large-scale reorganization of chromatin during the floral transition in Arabidopsis. Plant J 50:848-857

Weiss H, Maluszynska J (2000) Chromosomal rearrangement in autotetraploid plants of Arabidopsis thaliana. Hereditas 133: 255-261

Yang X, Timofejeva L, Ma H, Makaroff CA (2006) The Arabidopsis SKP1 homolog ASK1 controls meiotic chromosome remodeling and release of chromatin from the nuclear membrane and nucleolus. J Cell Sci 119:3754-3763

Zhang W, Lee HR, Koo DH, Jiang J (2008) Epigenetic modification of centromeric chromatin: hypomethylation of DNA sequences in the CENH3-associated chromatin in Arabidopsis thaliana and maize. Plant Cell 20:25-34 